\title{
First direct observation of the tau neutrino
}

\author{
Thomas Patzak for the DONUT Collaboration
}

\section{History}

$T^{\mathrm{n}} 1930 \mathrm{~W}$. Pauli, in a famous letter to his colleagues, postulated the existence of a new particle - the neutrino. Pauli presented the neutrino as an explanation of the observation that the decay of the neutron produced a continuous $B$-spectrum. Since then, great progress has been made in particle physics to describe the most fundamental constituents and interaction of matter. Nevertheless, many fundamental questions about neutrinos need to be answered. For example: Are neutrinos massive? Do they have a magnetic moment? Do they oscillate? Does a sterile neutrino exist? Is there direct evidence for the existence of the tau neutrino? The last question was finally answered in July 2000 by a group of 52 physicists from the DONUT Collaboration (Fermilab experiment E872) at Fermilab near Chicago.

The tau neutrino was postulated in 1975 after the discovery of the tau lepton by M. L. Perl et al. as the third generation neutrino in the Standard Model of electroweak interactions [1].

Glashow, Salam and Weinberg proposed the Standard Model in the late 1960s as a unified theory of the electromagnetic and weak interactions based on the gauge group $S U(2) \times U(1)$. This model has been successful in describing all recent experimental observations. Neutrinos enter this model as massless, neutral fermions. From measurements of the $\mathrm{Z}^{0}$ decay width, it was determined that there are three light neutrinos in nature. The charged and neutral leptons in the Standard Model are represented by three left-handed doublets of weak isospin.

\begin{tabular}{|c|c|c|c|}
\hline $\mathrm{Q}$ & $\mathrm{L}_{\mathrm{e}}=1$ & $\mathrm{~L}_{\mu}=1$ & $\mathrm{~L}_{\tau}=1$ \\
\hline-1 & $\mathrm{e}^{-}$ & $\mu^{-}$ & $\tau^{-}$ \\
\hline 0 & $\mathrm{v}_{\mathrm{e}}$ & $\mathrm{v}_{\mu}$ & $\mathrm{v}_{\tau}$ \\
\hline
\end{tabular}

Each flavor doublet carries a lepton flavor number $\left(L_{\text {e, }}\right.$ $\mathrm{L}_{\mu}, \mathrm{L}_{\tau}$ ) which is a conserved quantum number in the Standard Model. The DONUT experiment established the existence of the tau neutrino in the same manner as the two other neutrino flavors have been detected - by its charged current interaction.

\section{The Experiment}

The DONUT experiment (Direct Observation of NUTau) was built at Fermilab to observe the charged current interactions of $v_{\tau}$ and its charge conjugate. These interactions are identified by the detection of the tau lepton as the only lepton created at the primary vertex. The tau has a lifetime of $2.9 \times 10^{-13} \mathrm{~s}$. At the beam energy of the DONUT experiment the tau typically decays within $2 \mathrm{~mm}$ from the vertex into a single charged particle and neutrinos:

$$
\begin{gathered}
v_{\tau}+N \rightarrow \tau^{-}+X \\
\tau \rightarrow\left(\mu^{-} \text {or } e^{-}\right) v_{\mu} v_{\tau} \text { or } \tau^{-} \rightarrow h^{-} v_{\tau}
\end{gathered}
$$

The characteristic signature of a tau neutrino event is the observation of a primary interaction track with a bend point or kink, identifying this track as a tau lepton.

Nuclear emulsions were used as high resolution tracking devices in this experiment.

The resolution achieved with emulsions is better than $1 \mu \mathrm{m}$. The emulsion target was followed by a spectrometer which was used to determine the charge, energy and particle identification of the decay products. A schematic of the experiment is shown in Figure 1.

\section{The Neutrino Beam}

The neutrino beam used by the experiment was produced by 800 $\mathrm{GeV}$ protons from the Fermilab Tevatron interacting in a onemeter long tungsten beam dump. The principal source $(\sim 85 \%)$ of tau neutrinos and tau anti-neutrinos is the leptonic decay of the charmed meson $D_{s}$, into $\tau$ and $v_{\tau}$, and the subsequent decay of the $\tau$ to $v_{\tau}$. All charged particles produced in the dump were swept away from the emulsion target region by magnets or were absorbed by concrete, iron and lead shielding.

\section{The Detector}

The DONUT detector consisted of a scintillation counter veto wall, emulsion target, trigger hodoscopes, analysing magnet, drift chambers, calorimeter and muon identifier. We used two different types of emulsion targets. The first type called "ECC" (Emulsion Cloud Chamber), was composed of $1 \mathrm{~mm}$ thick stainless steel plates interleaved with emulsion plates. These plates were composed of $100 \mu \mathrm{m}$ thick emulsion layers on either side of a $200 \mu \mathrm{m}$

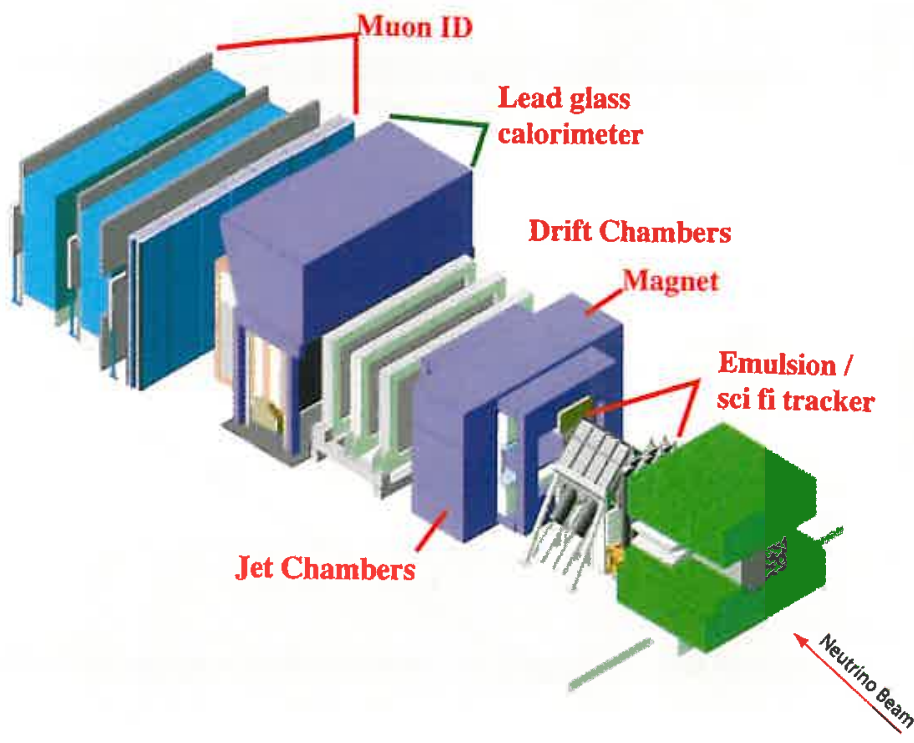

Fig. 1: Schematic view of the DONUT detector. 


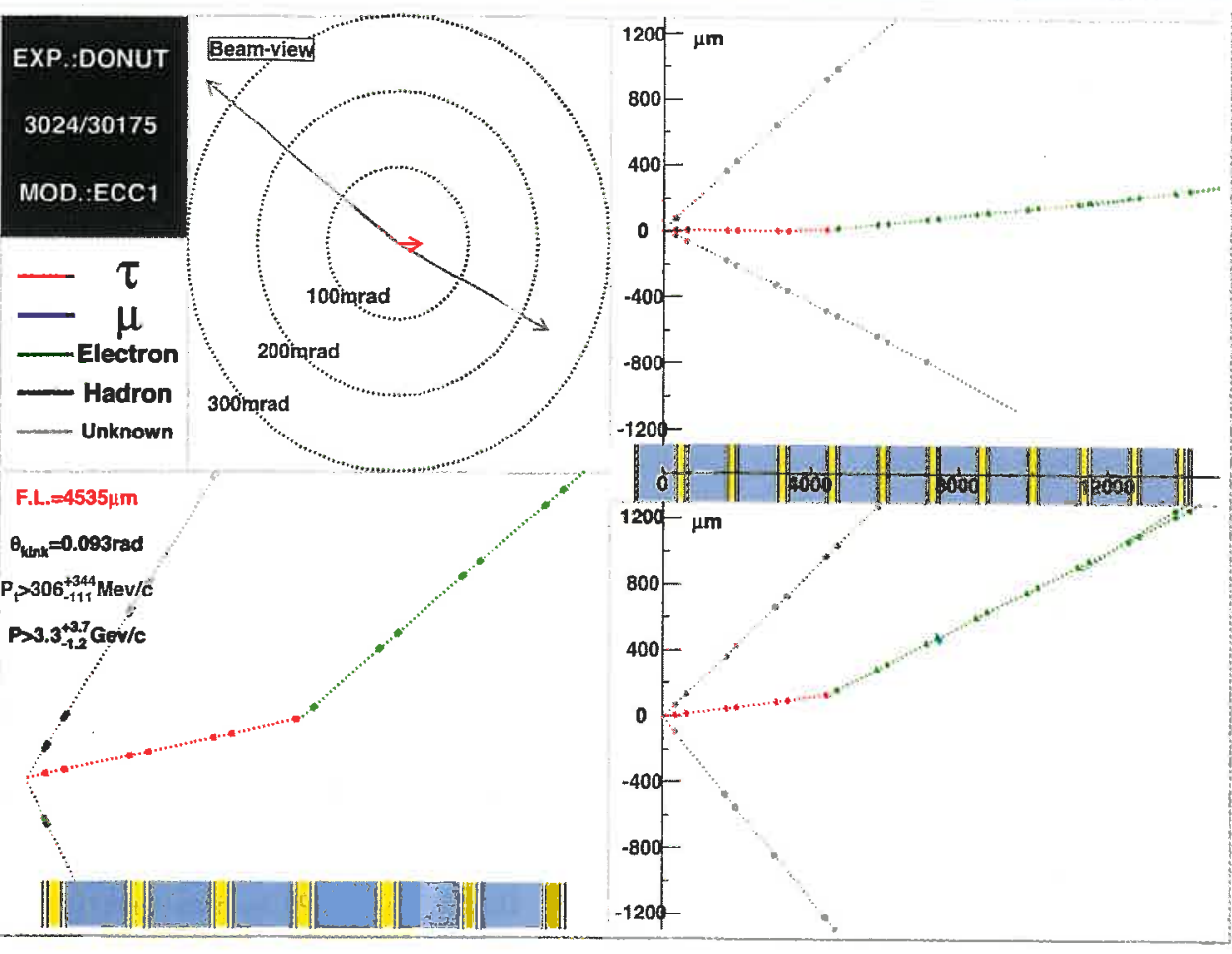

Fig. 2: One of the four observed tau neutrino charged current interactions. The kink signature of the tau decay is clearly visible. The target is represented at the bottom: steel $=$ blue emulsions $=$ yellow In this example the tau decays into an electron. or $800 \mu \mathrm{m}$ thick plastic base. A target module consisted of $\sim 50$ steel/emulsion layer pairs to obtain a thickness of 0.16 interaction lengths. The bulk type was composed exclusively of emulsion plates with $350 \mu \mathrm{m}$ thick emulsion layers on either side of a $90 \mu \mathrm{m}$ thick plastic base. These modules were 0.13 interaction lengths thick. The transverse size of all emulsion and steel sheets was $50 \mathrm{~cm} \times 50 \mathrm{~cm}$. Between emulsion modules, 44 planes of scintillating fibers provided precision tracking, and were read out by an image intensifier system.

\section{The Event Analysis}

Neutrino interactions in the emulsion target were selected by trigger hodoscopes in the target region. For each accepted event all electronic detector components were read out. The information from the spectrometer and calorimeter was used to select possible candidate events. All charged particle tracks from these candidate events were projected back to the exit point of the emulsion target using the scintillating fiber tracking system. The reconstructed tracks were then used to reconstruct a vertex with a typical precision of $1 \mathrm{~mm}$ in the transverse coordinate and $7 \mathrm{~mm}$ in the beam direction. This event selection was used to limit the size of the search volume in the emulsion target. During the run, $4.0 \times 10^{6}$ triggers were recorded from $3.54 \times 10^{17}$ protons incident on the tungsten target. After further cuts on the event geometry and energy a total of 898 events were classified as neutrino interaction candidates. Of the 898 candidates, 698 had a vertex predicted within the emulsion fiducial volume. Additional requirements on the event topology and vertex precision reduced the sample to 499 events. These 499 events were scanned using a fully automated emulsion scanning stations, which followed all tracks from the vertex through the different emulsion sheets. The result of this scanning is a 3D digitised image of the interaction with a precision better than $1 \mu \mathrm{m}$. A valid vertex was located for 262 of the 499 events.

A method of fully automated scanning has been developed over the past 20 years by the group of K. Niwa and his colleagues in Nagoya, Japan $[2,3]$. This technique was used in the CHORUS experiment, and most recently allowed the direct observation of the $v_{\tau}$ in DONUT. The capacity to analyse a large volume of emulsion will allow the use of massive targets of nuclear emulsion in future high-energy experiments.

\section{The Result}

Emulsion plates containing located events were rescanned within an adjusted scanning volume centred on the event. After further alignment 203 events satisfied the criterion of a track resolutions better then $0.6 \mu \mathrm{m}$. In these 203 events a detailed decay search was performed, looking for the event topology of a tau neutrino interaction - a kink in one of the primary tracks.

The selection criteria for $v_{\tau}$ events were established by Monte Carlo simulation.

After the application of these selection criteria to the sample of 203 events, four events remained. This experimental result is consistent with the number of expected $\tau$ events in the sample, 4.2 events. The total number of background events is estimated to be $0.34 \pm 0.05$. Therefore the probability that all four events are a result of a background fluctuation is $4 \times 10^{-4}$.

Figure 2 shows one of the four events, the first direct observation of charged current tau neutrino interactions. These events confirm the existence of $v_{\tau}$ as a partner of the tau lepton in the Standard Model of electroweak interactions.

For more detailed information on the event selection procedure see reference [4].

\section{References}

[1] M. L. Perl et al., Phys. Rev. Lett. 35, 1489 (1975).

[2] S. Aoki et al., Nucl. Instr. Meth. B51, 466 (1990).

[3] T.Nakano, Ph.D. Thesis, Nagoya University (1997).

[4] K. Kodama et al., Observation of Tau Neutrino Interactions, submitted to Phys. Lett. B. 